\title{
AH-AODV: Adaptive Hello Messaging based AODV Routing Protocol
}

\author{
Priyanka Thakkar \\ Computer Science Department \\ K.J.Somaiya College of Engineering \\ Mumbai, India
}

\author{
Prasanna Shete \\ Computer Science Department \\ K.J.Somaiya College of Engineering \\ Mumbai, India
}

\begin{abstract}
Ad hoc On-demand Distance Vector routing protocol (AODV) is a reactive routing protocol used in MANETs that makes use of Hello Messages for local Link connectivity. Every node in a network broadcasts hello messages to all its neighbours at a default hello interval of 1 second. However, this continuous broadcast leads to unnecessary energy consumption and energy utilization. This paper suggests an Adaptive Hello messaging based approach that modifies the Hello message broadcast mechanism of AODV. This approach makes the hello interval adaptive by making it directly proportional to event interval thereby suppressing unnecessary hello messages and breakage of links to the destination without adversely affecting the network performance in terms of throughput, end-to-end delay, PDR and jitter.
\end{abstract}

\section{General Terms}

Algorithms, Performance, Design.

\section{Keywords}

MANET, AODV, Hello messages, Hello Interval.

\section{INTRODUCTION}

The Ad hoc On-demand Distance Vector routing protocol (AODV) [1] is a reactive routing protocol used in MANETs. It offers quick adaptation to dynamic link conditions, low processing and memory overhead, low network utilization, and determines unicast routes to destination within the ad hoc network. AODV protocol operates 'On-demand' [2] i.e. its broadcasts routing information only to those neighbours which have an active route to the destination. It makes use of four control messages 1) RREQ (Route Request) messages

that are used for route discovery. Sender initiates the route discovery by broadcasting RREQ messages to all its neighbours.[9] When an intermediate node receives this RREQ message it forwards it to a node that has an active route to the destination, else rebroadcasts it to all its neighbours. This process increases the energy consumption as well as routing overhead thereby causing the broadcast storm problem [3]. 2) RREP (Route Reply) messages are generated by the node and sent to the source node in two cases, 1) If the node itself is the destination 2) If it has an active route to the destination. 3) The RERR (Route Error) message is sent whenever a link break causes one or more destinations to become unreachable from some of the node's neighbours. 4) Hello messages are used for local link connectivity i.e. every node broadcasts hello messages to all its neighbours to determine whether the neighbouring nodes are still within the range or alive [4]. A node broadcasts hello messages after every 1 second. The hello messages are redundant in case if there is a continuous communication taking place between the neighbouring nodes. The frequent broadcast of hello messages in such cases causes unnecessary bandwidth utilization and energy consumption. In order to suppress unnecessary hello messages, hello interval was made adaptive based on the event interval. The analysis and simulation of the proposed scheme suppresses the number of hello messages sent by $22.25 \%$ without adversely affecting the network performance.

The rest of the paper is organized as follows. Survey of different optimization approaches is presented in section 2 . Adaptive Hello messaging based AODV routing protocol (AH-AODV) is proposed in section 3. Sections 4 and 5 present the simulation environment, and performance evaluation of the proposed algorithm. Conclusion is presented in section 6 .

\section{RELATED WORK}

The performance of traditional AODV protocol mainly degrades due to the routing overhead caused during the route discovery by the RREQ packets as well as due to the continuous hello messages broadcast. To optimize the routing mechanism of AODV various approaches are proposed [5]. Different optimization approaches are discussed here.

In order to solve the 'broadcast storm' problem in AODV protocol a hybrid flooding scheme is suggested in [7]. It combines the features of probabilistic, neighbour based and area based flooding schemes. It makes use of density of nodes by using a density metric known as expansion metric. (Expansion metric determines how dense the 2 hop neighbourhood of a node is). Based on this value, forwarding probability of RREQ packets is adjusted. If this value is high, i.e. if the area is dense, the RREQ packets are flooded with low probability to reduce the redundancy of control packets, whereas in less dense areas RREQs are flooded with high probability. It also defines a forwarding zone that restricts the nodes by forwarding the RREQ packets if it lies outside the predefined zone. This scheme reduces the routing overhead and energy consumption as compared to the simple flooding carried out by AODV protocol during route discovery.

Routing overhead increases due to broken links to the destination. In order to solve this problem, a Hybrid Location based Ad hoc routing protocol (HLAR) is proposed in [8]. HLAR makes use of expected transmission count (ETX) metric in order to find the best quality route to the destination. During route discovery, the sender puts the location coordinates of itself as well as the destination in the RREQ packet and then determines whether it has any closer neighbour to the destination or not. If yes, it forwards the packet to the closer neighbour else broadcasts it to all the neighbouring nodes. It allows the intermediate node to 
locally repair the link if it comes across any link breakage. The analysis and simulation of this protocol reduces the routing overhead considerably.

Very few attempts have been made to reduce the overhead incurred due to frequent broadcasting of hello messages. One such attempt was made to suppress unnecessary hello messages, is suggested in [5]. It proposes an adaptive hello interval based on the event interval in order to reduce the battery drain thereby reducing network overhead and hidden energy consumption. Simulation results of this scheme could reduce the energy consumption up to $54 \%$ without any additional delay.

\section{ADAPTIVE HELLO MESSAGING BASED AODV ROUTING PROTOCOL (AH-AODV)}

The traditional AODV protocol uses Hello messages for local link connectivity by broadcasting them at a default hello interval of 1 second. However these hello messages do not have a significant role in case there is a continuous communication taking place between the two nodes and hence, there is an unusual consumption of the bandwidth and energy. In order to optimize the performance of traditional AODV protocol, Adaptive Hello messaging based AODV (AH AODV) routing protocol was used, which suppresses unnecessary broadcasting of Hello messages. This approach explains how unnecessary hello messages can be suppressed without any considerable difference in the Quality of Service (QoS) parameters like Throughput, End-to-End Delay and Packet Delivery Ratio etc. For this hello interval was made adaptive by making it directly proportional to event interval. In a network, an event can be sending and receiving data packets between the source node and the destination node, sending Route Reply (RREP) messages from the destination back to the source, sending Route Reply (RREP) messages from an intermediate node to the originator of RREQ message and receiving RREP messages by either the source node or an intermediate node. These events occur on an active route that has been established using a route discovery mechanism between the source node and the destination node. The time of each of the above mentioned events was first recorded and then the event interval was calculated. 'Event Interval' is the time gap between the number of packets sent (RREP, data) or received (RREP, Data) by a particular node. Depending on this Event Interval, Hello interval is varied thereby making it adaptive. A 'Neighbour table' was generated to store information like neighbour source address for a particular node, type of the packet sent/ received by a particular node, time at which these packets were sent/received and the calculated Event interval. In order to calculate the event interval, two structures were created, neighbour table and buffer table. If a node with node identifier ' $x$ ' performs any of the above mentioned events at time $\mathrm{t} 1$, its neighbour table is displayed in the output window and this neighbour table is stored in the buffer table. This neighbour table is refreshed after every 6 seconds. When the same node with node identifier ' $x$ ' participates in any of the said events at time $\mathrm{t} 2$, Event interval for node with identifier ' $\mathrm{x}$ ' is calculated as follows:-

Event Interval $(E I)=\mathrm{t} 1$ (Stored in neighbour table $)-\mathrm{t} 2$ (Stored in buffer table)

Based on this Event Interval, Hello interval is varied.

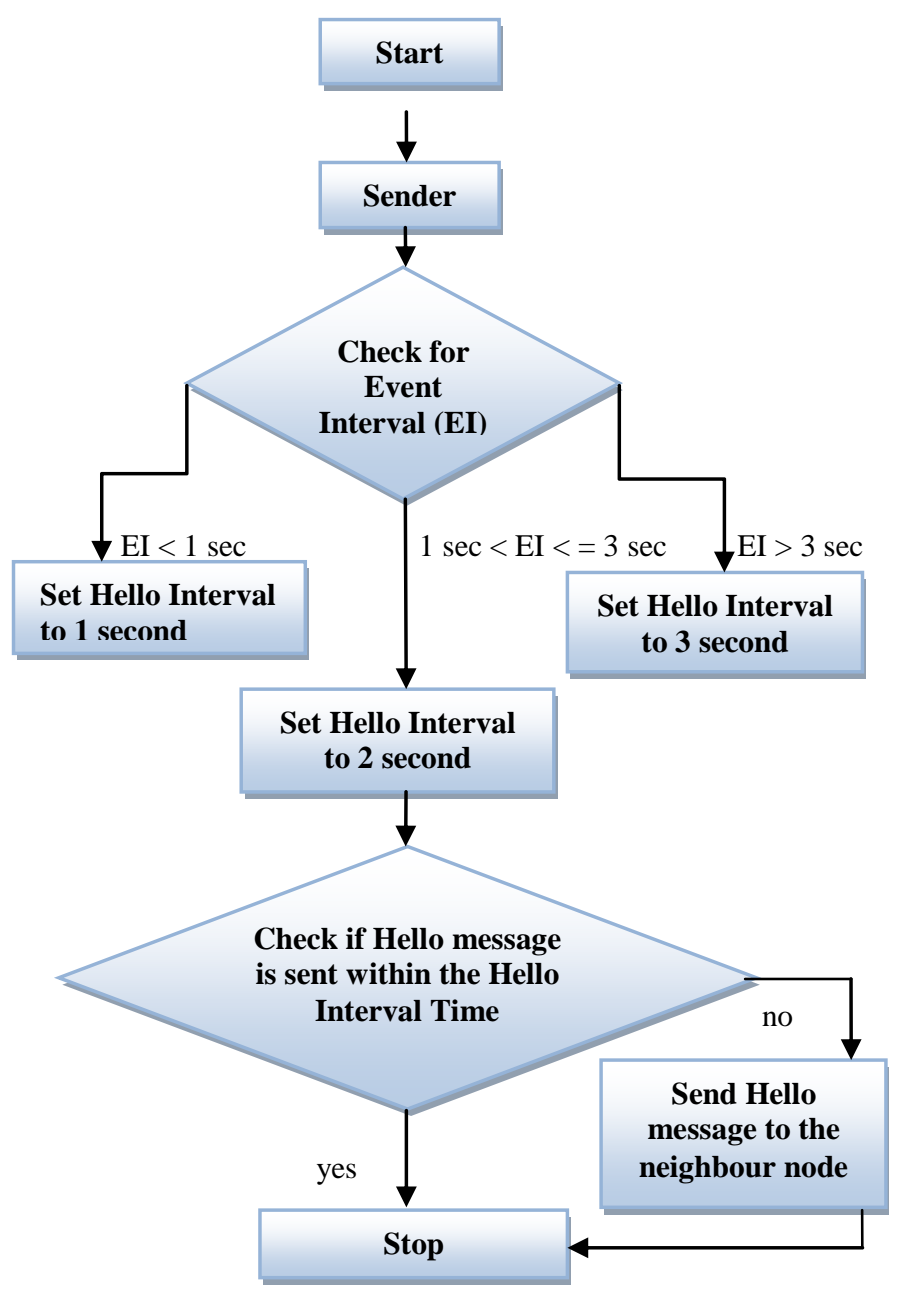

Figure 1. Flowchart of AH-AODV

When the event interval does not satisfy any of the three domains, hello interval was set to a static value of 2.5 seconds in the following experimentation.

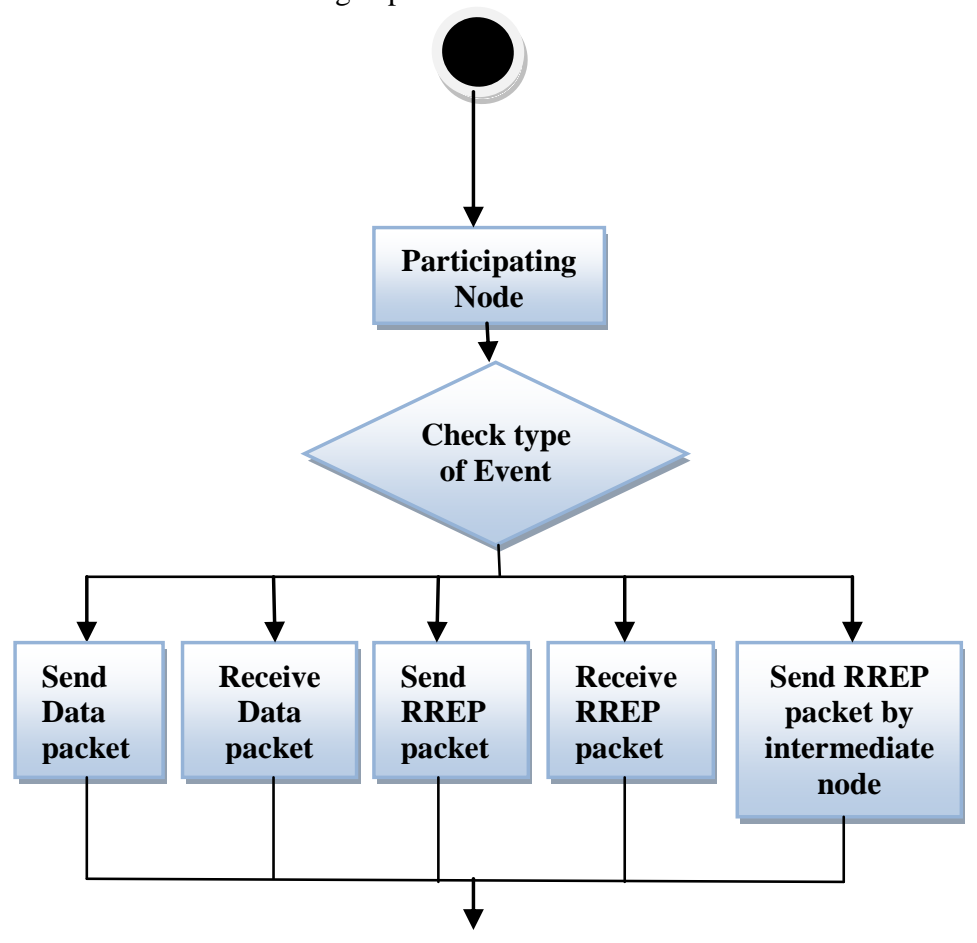




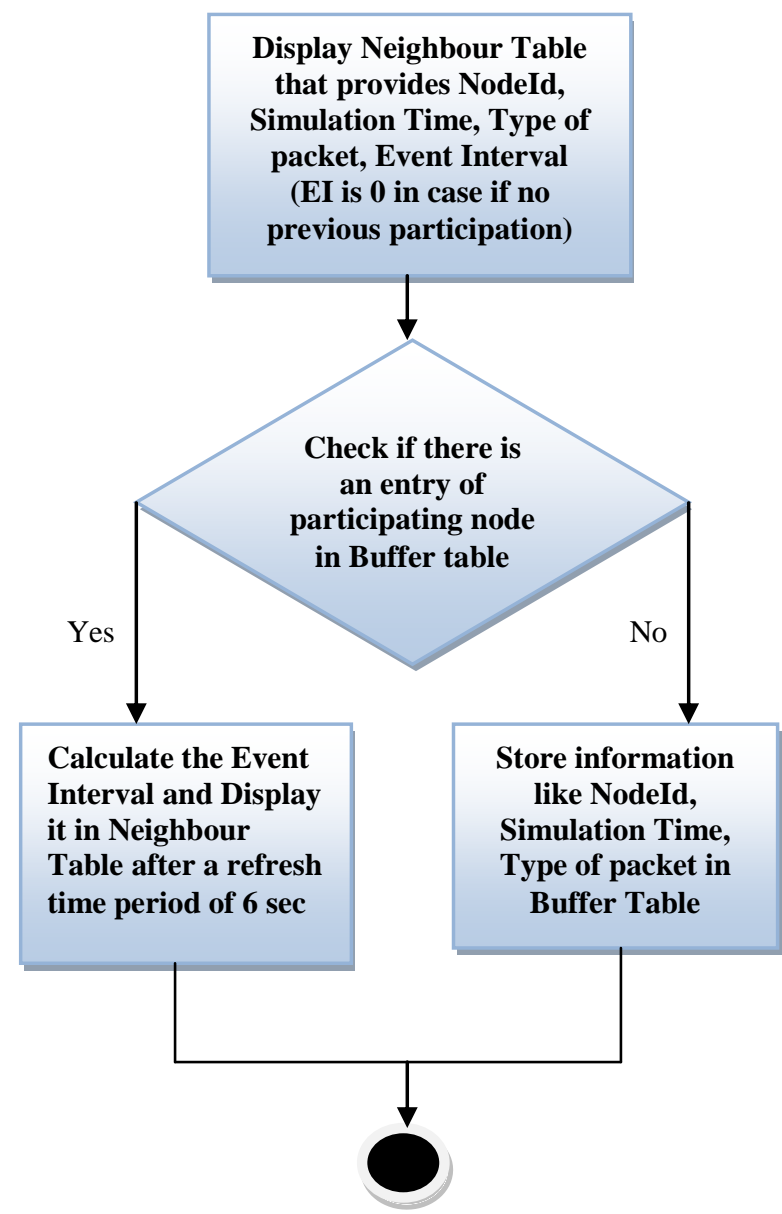

Figure 2. Working of AH-AODV

\section{PERFORMANCE EVALUATION}

QualNet 5.0.2 Network Simulator was used to perform experiments and validate the proposed scheme [10]. For each and every scenario, the number of nodes is set to 50 . The traffic used in each of the scenario between the source and the destination is CBR (Constant Bit rate). The performance of AH AODV protocol and the traditional AODV protocol was compared in terms of throughput, End-to-End Delay, Jitter, Packet Delivery Ratio, Number of times the link broke and the number of Hello messages sent by each node.

\section{Simulation environment 1}

Table 1. Simulation parameter 1

\begin{tabular}{|l|l|}
\hline Parameter & Value \\
\hline No. of Nodes and Area & $\begin{array}{l}50 \text { nodes } \\
1000 \mathrm{~m} * 1000 \mathrm{~m}\end{array}$ \\
\hline $\begin{array}{l}\text { Node Placement } \\
\text { Strategy }\end{array}$ & Random Waypoint \\
\hline Simulation Time & 3 minutes \\
\hline Channel Frequency & $2.4 \mathrm{GHz}$ \\
\hline
\end{tabular}

\begin{tabular}{|l|l|}
\hline Path Loss Model & Two ray Model \\
\hline Transmission Range & $350 \mathrm{~m}$ \\
\hline $\begin{array}{l}\text { PHY / MAC Layer } \\
\text { Protocol }\end{array}$ & IEEE $802.11 \mathrm{~b}$ \\
\hline Speed & $10 \mathrm{~m} / \mathrm{s}$ \\
\hline Traffic Sending Rate & $\begin{array}{l}16,32,64,128,256,512,102 \\
4 \mathrm{kbps}\end{array}$ \\
\hline Payload size & 512 bytes \\
\hline
\end{tabular}

Initially the performance of AH-AODV (in terms throughput, end-to-end delay, PDR and Jitter) is same as that of traditional AODV, since the network is operated below channel capacity. However, as sender data rate increases beyond saturation limit, AH-AODV outperforms conventional AODV.

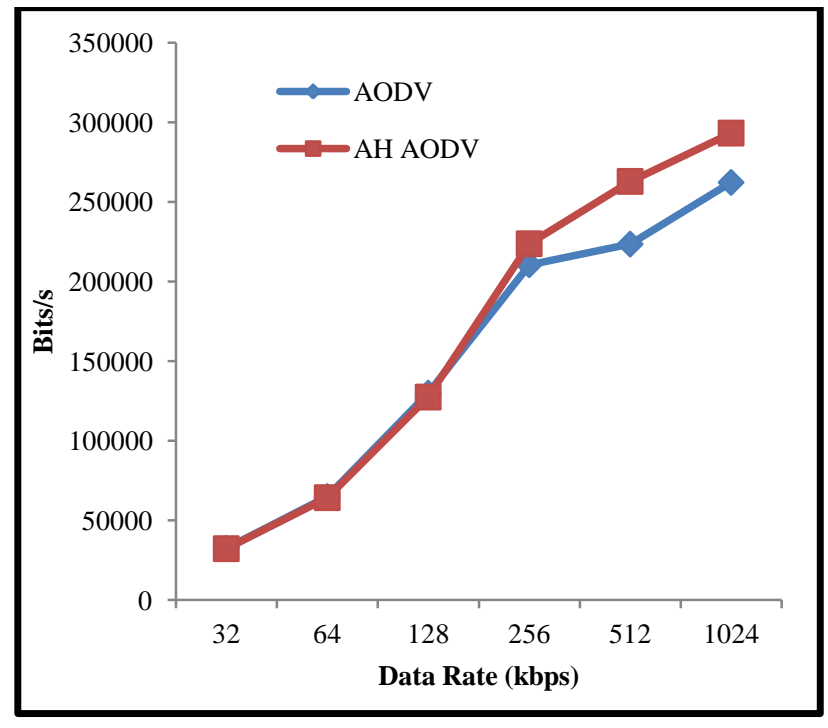

Figure 3. Throughput vs Data Rate

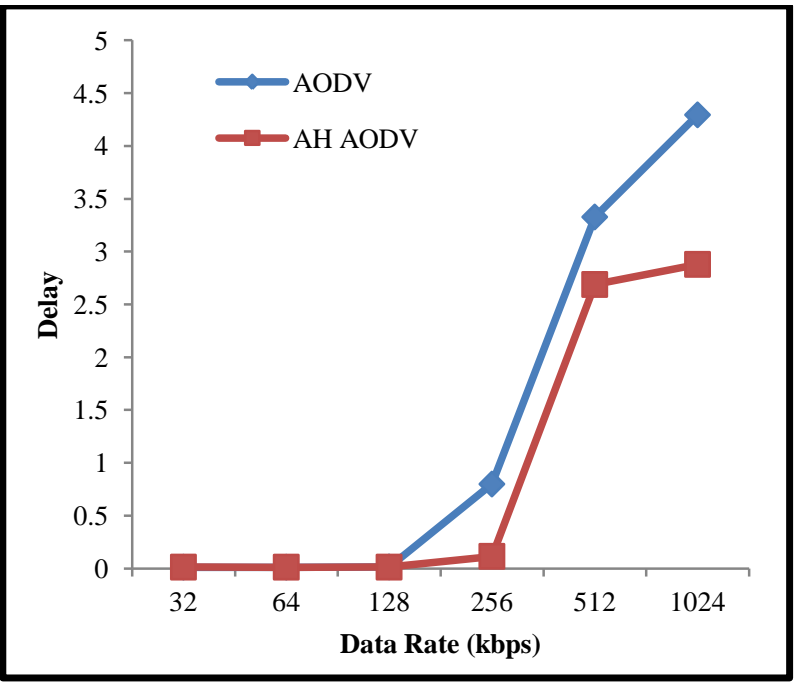

Figure 4. End to End Delay vs Data Rate 


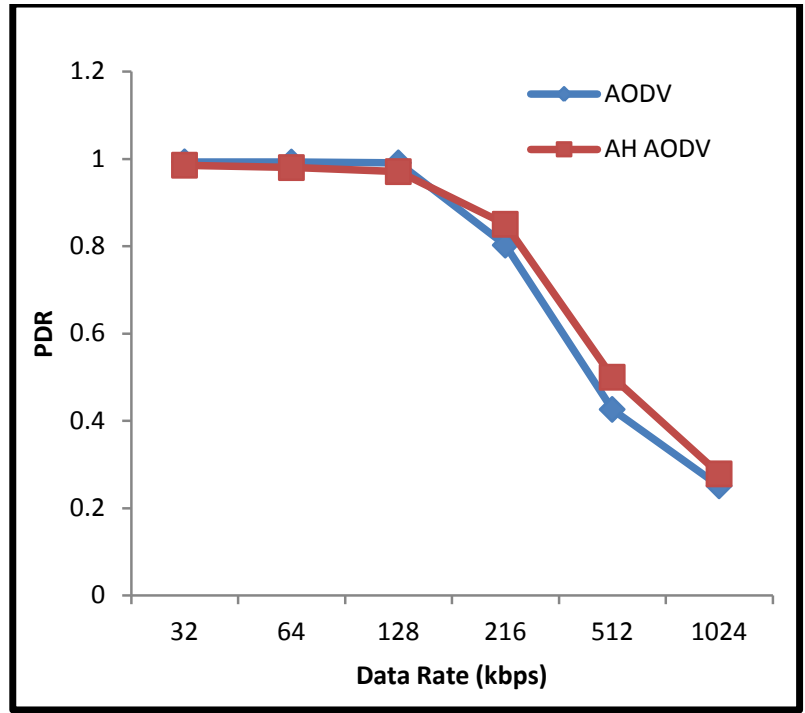

Figure 5. Packet Delivery Ratio vs Data Rate

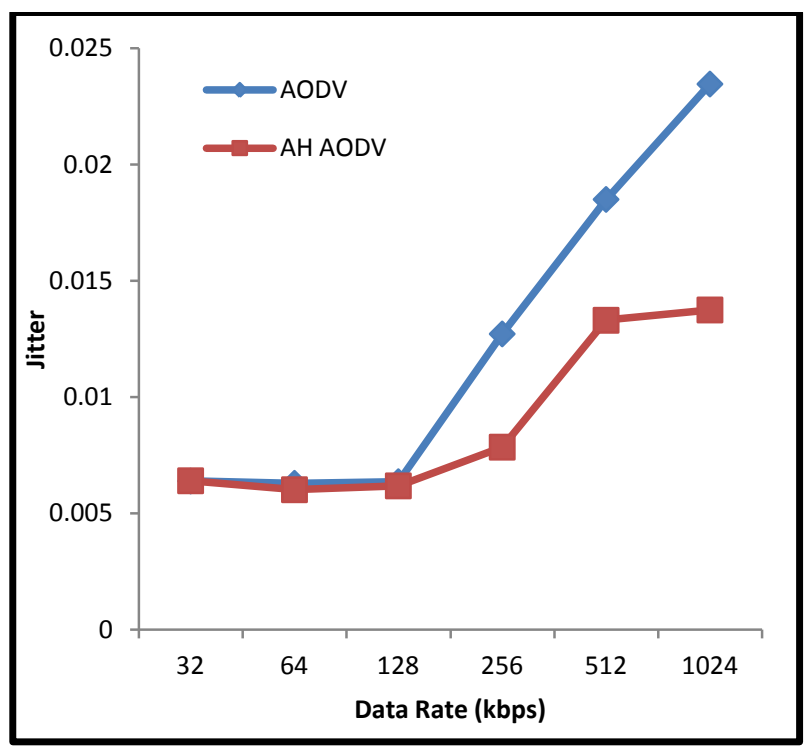

Figure 6. Jitter

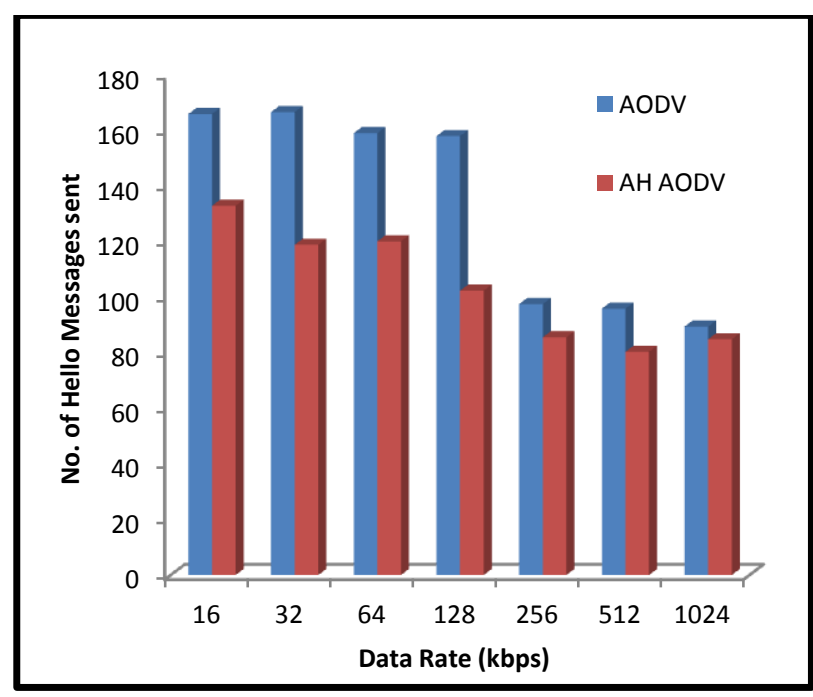

Figure 7. Number of Hello messages sent

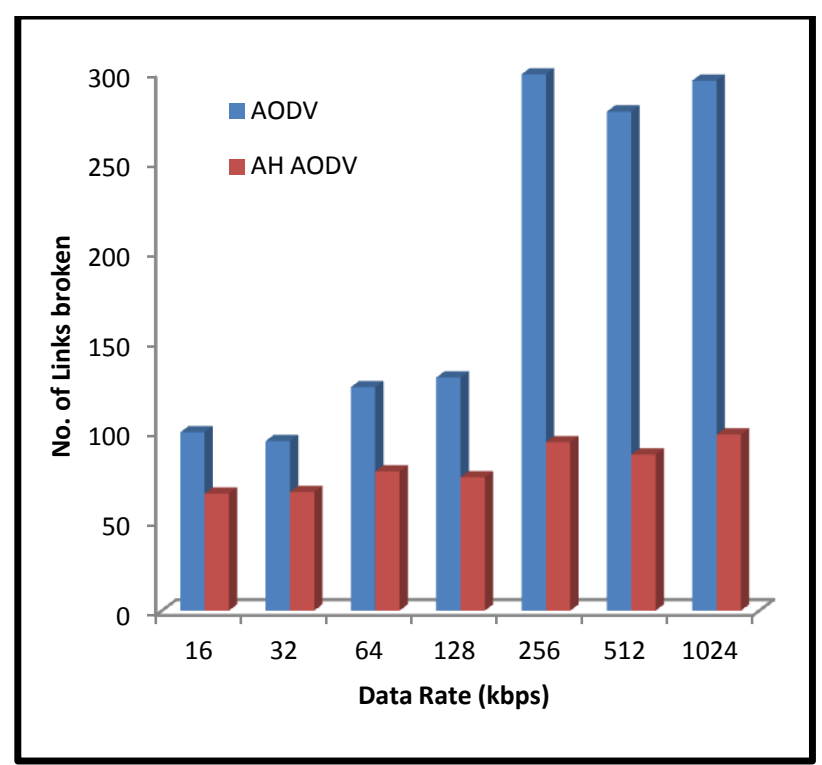

Figure 8. Number of times Link broke

Simulation environment 2

In this section, the performance of AH AODV protocol was analysed and compared with the traditional AODV protocol by keeping the data rate constant at $512 \mathrm{kbps}$ and varying the speed. The simulation environment can be summarized in terms of a table as shown below.

Table 2. Simulation parameter 2

\begin{tabular}{|l|l|}
\hline Parameter & Value \\
\hline $\begin{array}{l}\text { No. of Nodes and } \\
\text { Area }\end{array}$ & $\begin{array}{l}50 \text { nodes } \\
1000 \mathrm{~m} * 1000 \mathrm{~m}\end{array}$ \\
\hline $\begin{array}{l}\text { Node Placement } \\
\text { Strategy }\end{array}$ & Random Waypoint \\
\hline Simulation Time & 3 minutes \\
\hline Channel Frequency & 2.4 GHz \\
\hline Path Loss Model & Two ray Model \\
\hline Transmission Range & $350 \mathrm{~m}$ \\
\hline $\begin{array}{l}\text { PHY / MAC Layer } \\
\text { Protocol }\end{array}$ & IEEE $802.11 \mathrm{~b}$ \\
\hline Speed & $2.5,5,10,15,20,25 \mathrm{~m} / \mathrm{s}$ \\
\hline Traffic Sending Rate & $512 \mathrm{kbps}$ \\
\hline Payload size & $512 \mathrm{bytes}$ \\
\hline
\end{tabular}




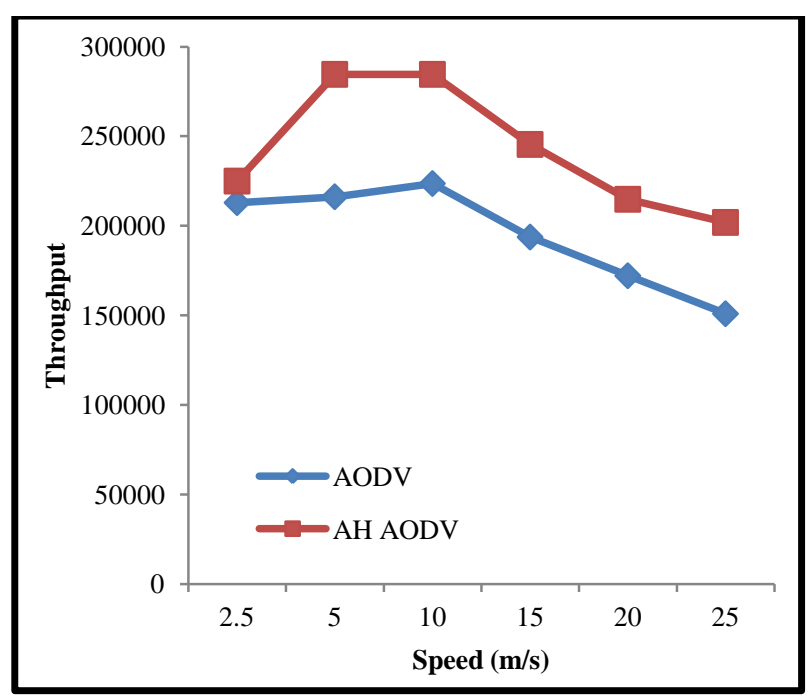

Figure 9. Throughput vs Speed

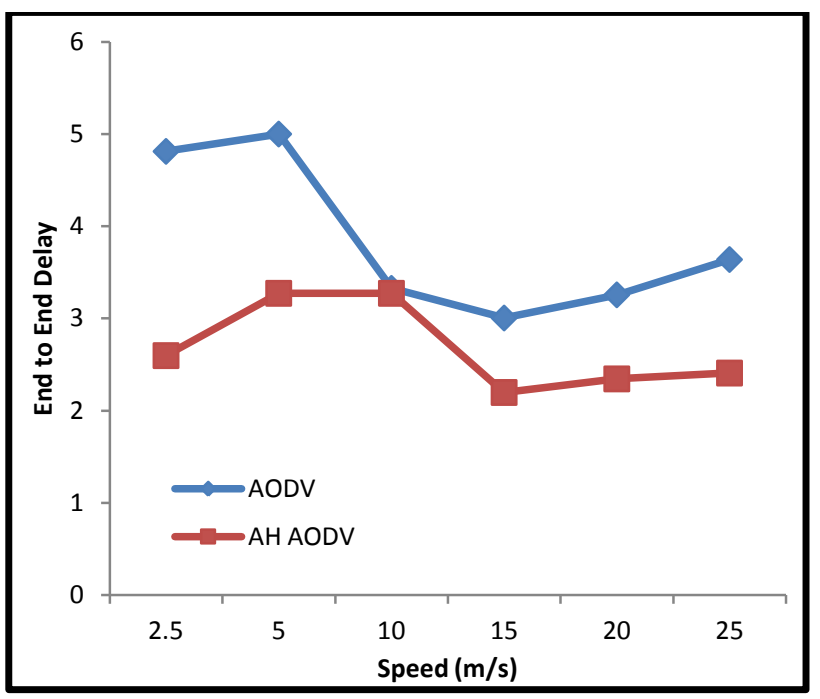

Figure 10. End to End Delay vs Speed

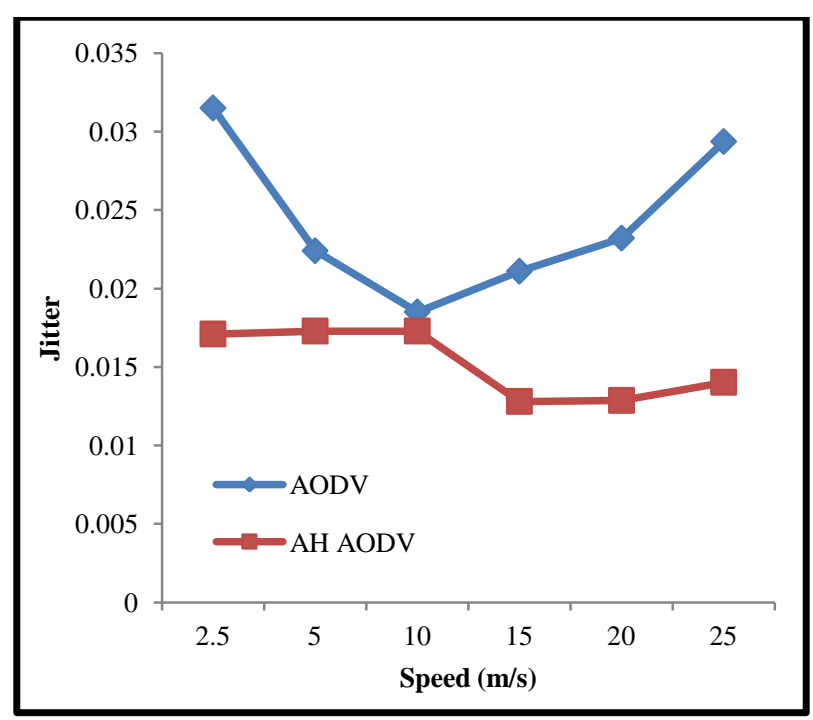

Figure 11. Jitter vs Speed

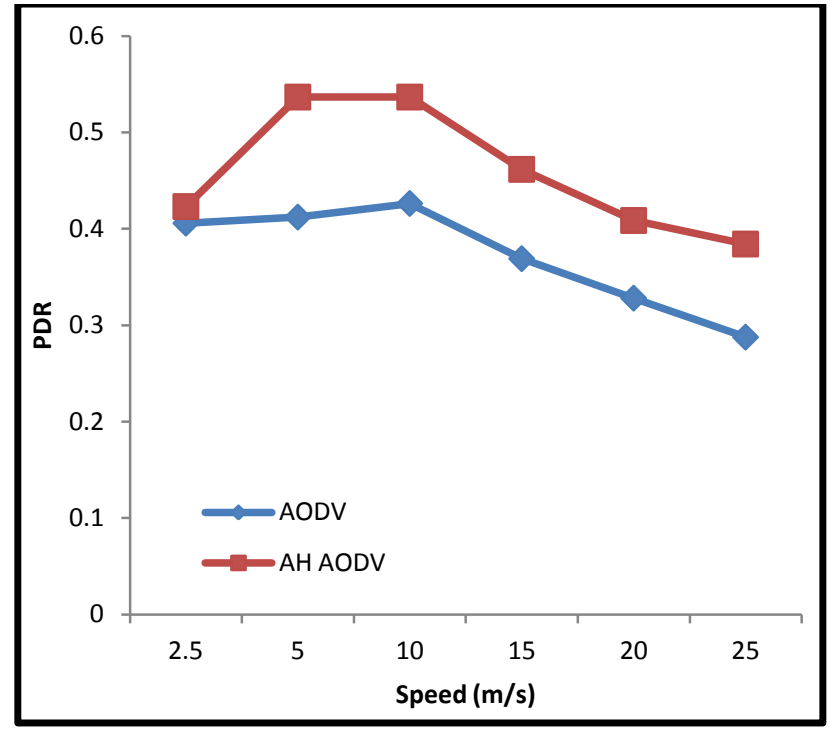

Figure 12. Packet Delivery Ratio vs Speed

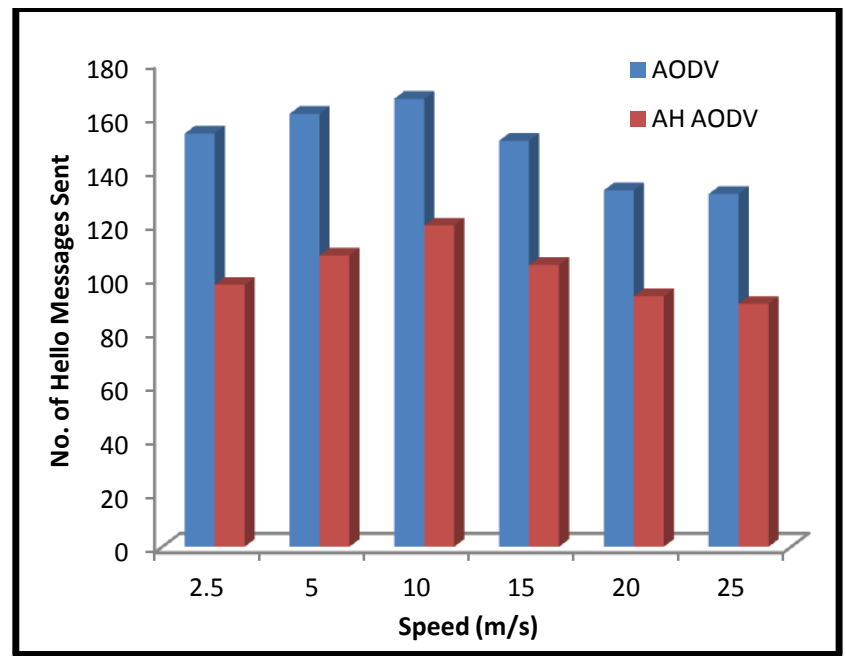

Figure 13. Number of Hello messages sent

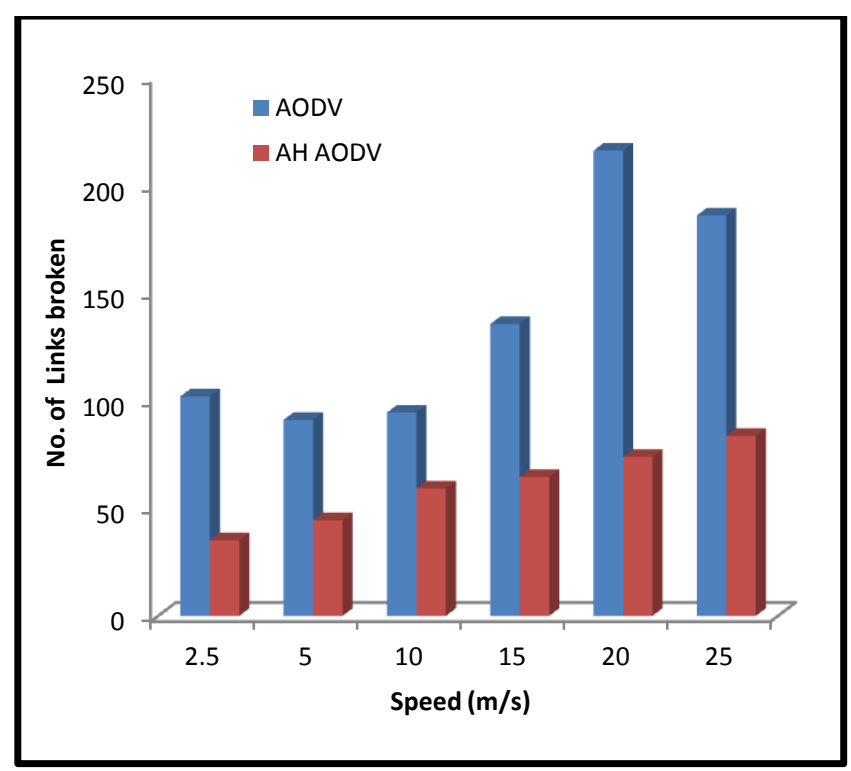

Figure 14. Number of times Link broke 
The above simulations show that AH-AODV outperforms the conventional AODV protocol in terms of throughput, end-toend delay, packet delivery ratio and jitter. Also, it suppresses hello message broadcast by $30.57 \%$ without adversely affecting the network performance. Number of times the link to the destination broke also reduced considerably thereby avoiding unnecessary routing overhead.

\section{CONCLUSION}

In this paper, an" Adaptive Hello messaging based AODV routing protocol (AH-AODV)" is proposed. The proposed scheme varies the hello interval based on the event interval thereby suppressing unnecessary hello messages. Thus by saving the bandwidth utilization it improves the network performance in terms of throughput, end-to-end delay, PDR, jitter and suppresses broken links to the destination as well as number of hello messages broadcasted thus outperforming the conventional AODV protocol.

\section{REFERENCES}

[1] C. Perkins and S.das "Ad Hoc On Demand Distance Vector Routing (AODV) Routing Request For Comments (RFC) July 2003.

[2] C. E. Perkins, E. M. Royer, "Ad Hoc On-Demand Distance Vector Routing", Proceedings of IEEE Workshop on Mobile Computing Systems and Applications, pp. 90-100, February 1999.

[3] O. Tonguz, N. Wisitpongphan, J. Parikh, F. Bai, P. Mudalige and V. Sadekar, "On the Broadcast Storm Problem in Ad hoc Wireless Networks", Proc. of 3rd
Intl. Conf. on Broadcast Communications, Networks and Systems (BROADNETS), pp.1-11, Oct. 2006.

[4] Nisha Bhanushali, Priyanka Thakkar, Prasanna Shete, "Impact of Hello Interval on performance of AODV protocol", International Journal of Computer Applications, Volume 116-No. 23, April 2015.

[5] P. J. Shete, R. N. Awale, "Routing Protocols for Multihop Ad Hoc Networks: Operational Challenges and Design Optimizations", International Journal of Computer Applications, Vol. 84, No. 6, Dec 2013.

[6] S. Y. Han and D. Lee, "An Adaptive Hello Messaging Scheme for Neighbor Discovery in On-Demand MANET Routing Protocols", IEEE COMMUNICATIONS LETTERS, VOL. 117, N0. 5,pp. 1040-1043,MAY 2013.

[7] D. G. Reina, S. L. Toral, P. Jonhson, and F. Barrero, "Hybrid Flooding Scheme for Mobile Ad Hoc Networks", IEEE COMMUNICATIONS LETTERS, VOL.17, NO. 3, pp. 592-595, MARCH 2013.

[8] M. A Rabayah and R. Malaney, "A New Scalable Hybrid Routing Protocol for VANETs", IEEE Transactions on Vehicular Technology, Vol. 61, No. 6, pp. 2625-2635, July 2012.

[9] P. J. Shete, R. N. Awale, "RSS-Gossip AODV: Received Signal Strength based Gossip Flooding Mechanism for AODV", October 2014.

[10] User Manual QualNet Network Simulator, Version 5.0.2 